\title{
Highly sensitive curvature sensor based on asymmetrical twin core fiber and multimode fiber
}

\author{
Yue Wu ${ }^{a, b, *}$, Li Pei ${ }^{a, b}$, Wenxing Jin ${ }^{a, b}$, Youchao Jianga,b, Yuguang Yang ${ }^{a, b}$, \\ Ya Shen ${ }^{\mathrm{a}, \mathrm{b}}$, Shuisheng Jian ${ }^{\mathrm{a}, \mathrm{b}}$ \\ ${ }^{a}$ Key Lab of All Optical Network \& Advanced Telecommunication Network of EMC, \\ Beijing Jiaotong University, Beijing 100044, China \\ ${ }^{b}$ Institute of Lightwave Technology, Beijing Jiaotong University, Beijing 100044, China
}

\section{Abstract}

A highly sensitive curvature sensor based on asymmetrical twin core fiber (TCF) and multimode fiber (MMF) is proposed and experimentally demonstrated. By applying the coupled-mode theory and equivalent refractive index model, we theoretically analyze the uncoupled feature of the TCF and the relationship between peak wavelength and the curvature. Two segments of MMF used as beam splitter and combiner are embedded on the two ends of the TCF, and the extinction ratio of the comb transmission spectrum is about $15 d B$. The experimental result shows that the curvature sensitivity of the sensor can be achieved as high as $103.35 \mathrm{~nm} / \mathrm{m}^{-1}$ ranging from $0.24 \mathrm{~m}^{-1}$ to $0.6 \mathrm{~m}^{-1}$, and the strain sensitivity is up to $-4.01 \mathrm{pm} / \mu \varepsilon$ in the range from $0 \mu \varepsilon$ to $1400 \mu \varepsilon$. The simultaneous detection of the curvature and strain can be realized. The temperature sensitivity is $0.431 \mathrm{~nm} /{ }^{\circ} \mathrm{C}$ in the range from

\footnotetext{
*Corresponding author. Tel: +86 18813045260; Fax: +86 010 51683625(office)

Email addresses: 14111015@bjtu.edu.cn (Yue Wu), lipei@bjtu.edu.cn (Li Pei), 13111011@bjtu.edu.cn (Wenxing Jin), 13111018@bjtu.edu.cn (Youchao Jiang), 13111012@bjtu.edu.cn (Yuguang Yang), 13111014@bjtu.edu.cn (Ya Shen), ssjian@bjtu.edu.cn (Shuisheng Jian)
} 
$40{ }^{\circ} \mathrm{C}$ to $70{ }^{\circ} \mathrm{C}$. This fiber sensor exhibits the advantages of low cost, easy and repeated fabrication, and high sensitivity.

Keywords: twin core fiber, multimode fiber, curvature, strain

\section{Introduction}

Optical fiber sensors have been paid special attention due to their specific advantages, such as flexibility, high sensitivity, compact size, low cost, and immunity to electromagnetic interference over traditional sensors. Therefore, they have been widely applied in many areas for the measurement of parameter, such as strain, temperature, vibration and displacement. In recent years, optical fiber curvature sensor has attracted a wide attention in fields of composite material structures, robot arms, and artificial limbs.

A number of optical fiber curvature sensors have been proposed based on different optical fiber devices, such as long period grating (LPG) [13], chirped fiber Bragg grating (CFBG), tilted-fiber Bragg grating (TFBG) $[4,5]$, superimposed grating [6], singlemode-multimode-singlemode (SMS) fiber based Mach-Zehnder interferometer (MZI) [7], two peanut-shape structures modal interferometer [8], tapered fiber based MZI [9], hollow-core fiber and core-offset structure based MZI [10, 11], and the interferometers based on photonic crystal fiber (PCF) or polarization maintaining PCF [12-15]. However, most of these fiber sensors have their own disadvantages. It is difficult to read the resonance wavelength exactly due to the broad transmission resonance features of the LPG, and the demand of fabrication of fiber grating is higher, which will increase the cost. In addition, The fabrication of the superimposed grating and TFBG are very complicated in manufacturing. For 
hollow-core fiber structure, the light intensity modulation curvature sensor is easily disturbed by the light source, which has poor stability. The two peanut-shape structure usually involve complicated fabrication techniques, which has the limit in application. The cost of the PCF and polarization maintaining PCF is very high, and the core-offset and taper structure is fragile and easy to break.

In this paper, we propose and experimentally demonstrate a highly sensitive curvature sensor based on asymmetrical TCF and MMF. By applying the coupled-mode theory, we theoretically find that the custom-designed TCF has the uncoupled feature due to the large core pitch, and two MMFs are fusion spliced on the two ends of the dual-core fiber, which are used as beam splitter and combiner to form the in-fiber MZI. The relationship between peak wavelength and the curvature is found by applying equivalent refractive index model. In the experiment the best sensitivity of curvature can be achieved as high as $103.35 \mathrm{~nm} / \mathrm{m}^{-1}$ ranging from $0.24 \mathrm{~m}^{-1}$ to $0.6 \mathrm{~m}^{-1}$, and the best sensitivity of strain is $-4.01 \mathrm{pm} / \mu \varepsilon$ in the range from $0 \mu \varepsilon$ to 1400 $\mu \varepsilon$. The simultaneous detection of the curvature and strain can be realized. Besides, the best temperature sensitivity is $0.431 \mathrm{~nm} /{ }^{\circ} \mathrm{C}$ in the range from $40{ }^{\circ} \mathrm{C}$ to $70{ }^{\circ} \mathrm{C}$.

\section{Sensor design and operation principle}

The custom-designed TCF is fabricated by the commercial modified chemical vapor deposition (MCVD) method. The cross-section of the fiber is shown in Fig.1. The outer diameter of the asymmetrical TCF is about 128 $\mu m$, and the diameter of the dual cores are $7.1 \mu m, 9 \mu m$,respectively. A 


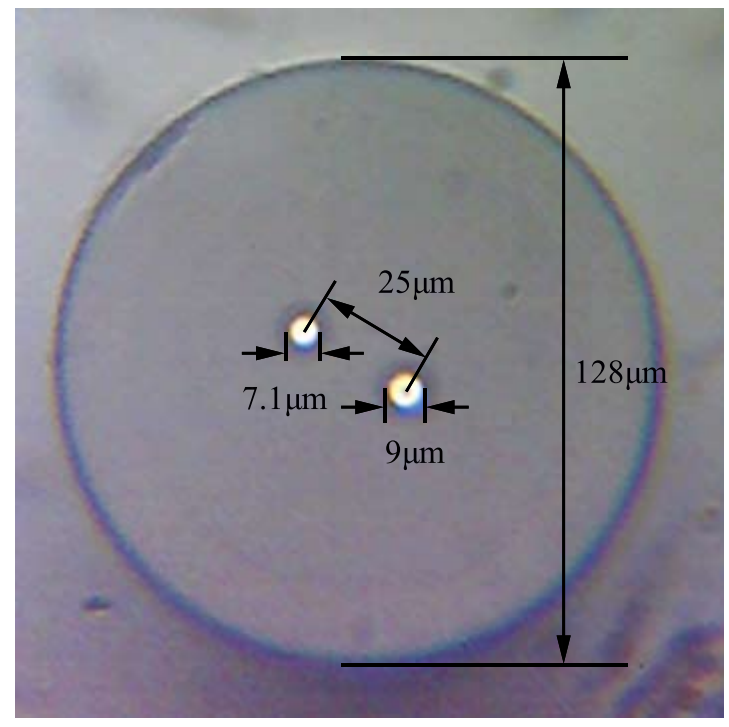

Figure 1: Micrograph of cross section of the TCF.

$0.35 \mathrm{~m}$ long TCF is used. The cores are originated from two Ge-doped Silica, which preforms with different diameters and the same refractive index, leading to asymmetrical index distribution in the horizontal direction. The refractive index difference $\Delta n$ of the core and the clad is $0.5 \%$. The distance between the dual cores is about $25 \mu \mathrm{m}$, which is enough to suppress the cross talk between the two cores. By applying the the coupled-mode theory, we theoretically analyze the relationship between the coupling coefficient and the distance between the dual cores. When the core pitch reaches to $25 \mu \mathrm{m}$, the TCF has no coupled feature as shown in Fig.2 (a). Meanwhile, we experimentally splice the TCF with single-mode fiber(SMF) by applying the lateral offset method. Fig.2 (b) shows the result, and we can not observe comb spectra.

Considering the uncoupled feature of the dual-core fiber, in the experi- 

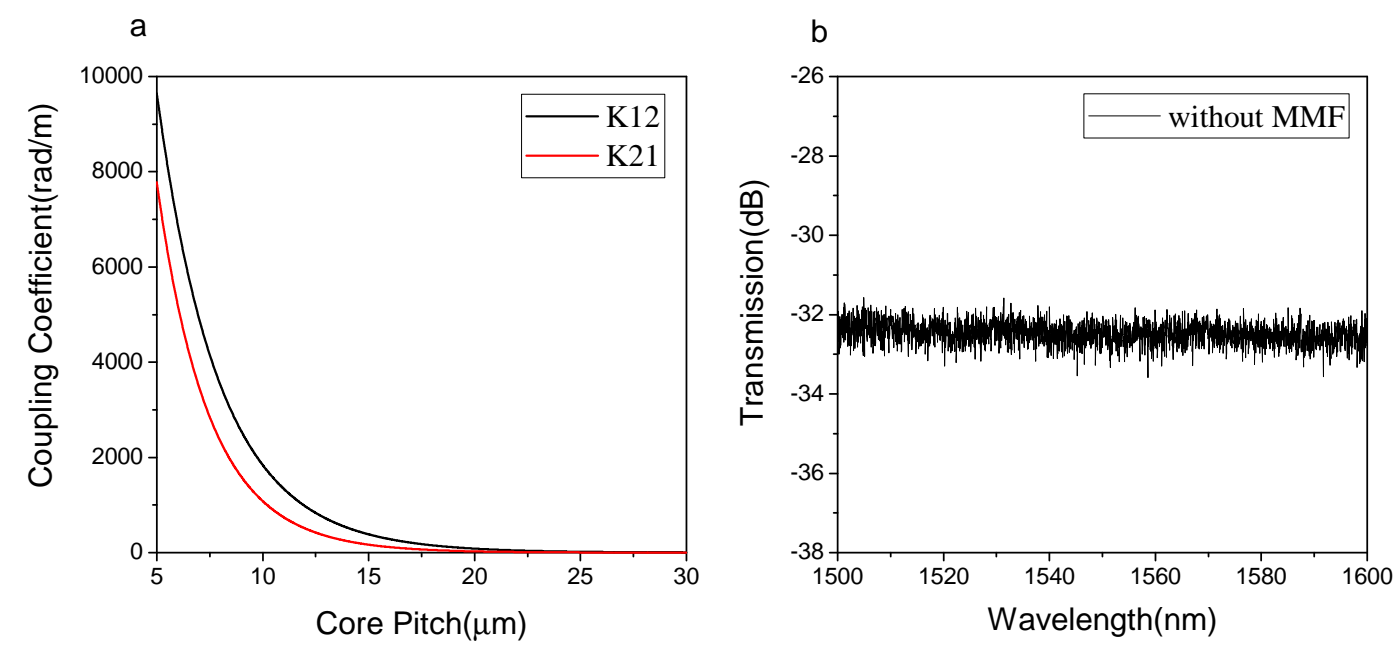

Figure 2: (a) Coupling coefficient against curvature; K12 (black line) and K21 (red line) are the coupling coefficient between core 1 and core 2. (b) Transmission spectrum of the TCF without MMFS.

ment two segments of MMF are fusion spliced on the two ends of the dualcore fiber, which are employed to achieve the beam splitting and combining. The outer diameter and core diameter of the MMF is $150 \mu m$ and $44 \mu m$,

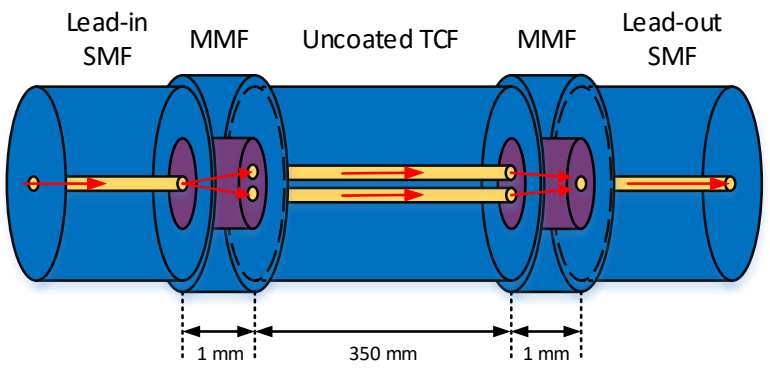

Figure 3: schematic diagram of the MZI filter.

respectively. A schematic of the MZI filter is illustrated in Fig.3. Due to that the core diameter of the MMF is larger than the core pitch of the TCF, when the power of singlemode fiber (SMF)-in reaches the first MMF, parts 
of power are coupled to dual cores. The two beams propagating in two cores are combined at the second MMF to form an in-fiber MZI. The transmitted light intensity of the MZI can be expressed by[16]

$$
I_{\text {total }}=I_{1}+I_{2}+2 \sqrt{I_{1} I_{2}} \cos \Delta \phi
$$

where $I_{\text {total }}$ is the transmitted light intensity of the MZI, $I_{1}$ and $I_{2}$ are the output powers of the dual cores of the TCF, respectively. $\Delta \phi$ is the phase difference between the two arms of the MZI which can be expressed as

$$
\Delta \phi=\frac{2 \pi L\left(n_{1}-n_{2}\right)}{\lambda}=\frac{2 \pi \Delta n L}{\lambda}
$$

where $\mathrm{L}$ is the length of the TCF, $\lambda$ is the vacuum wavelength. $n_{i}(\mathrm{i}=1$ or 2$)$ is the effective refractive index of the two cores, and $\Delta n$ is the effective refractive index difference between the two cores. When the phase difference $\Delta \phi$ is equal to $2 m \pi$, the output power $I_{\text {total }}$ of MZI filter reaches the maximum peak value which corresponds to the peak wavelength $\lambda_{m}$. The peak wavelength $\lambda_{m}$ can be deduced from Eq.(2).

$$
\lambda_{m}=\frac{\Delta n L}{m}
$$

Thus the difference of the adjacent peak wavelength can $\Delta \lambda$ be calculated as

$$
\Delta \lambda=\lambda_{m}-\lambda_{m+1}=\frac{\lambda_{m} \lambda_{m+1}}{\Delta n L} \approx \frac{\lambda_{m}^{2}}{\Delta n L}
$$

where $\Delta \lambda$ is the free spectral range (FSR).

Fig.4 (a) shows the schematic of bending TCF, $\mathrm{R}$ is curvature radius, and the origin of coordinates locates at the center position between the two cores. When the curvature is applied to the TCF, the outer layer of fiber 
a

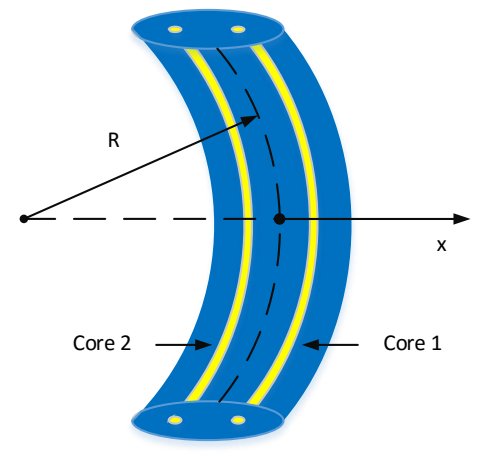

b

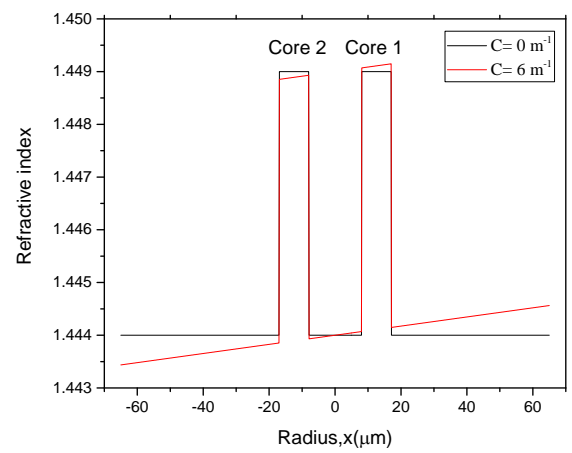

Figure 4: (a) Schematic of bending TCF. (b)Refractive index profile of the TCF .

(positive $\mathrm{x}$-direction) is in tension, and the inner layer (negative $\mathrm{x}$-direction) is in compression. According to elastic-optic effect, the refractive index of the outside layers becomes higher and that of the inside layers becomes lower. The refractive index profile in $\mathrm{x}$-direction can be expressed through equivalent refractive index model [17]

$$
n^{\prime}(x)=n(x)(1+C x)
$$

where $C$ is the curvature, $n^{\prime}(x)$ and $n(x)$ are refractive index profiles when the TCF is straight and bent, respectively. Fig.4 (b) shows that when the TCF is bent, the refractive index of core 1 would increase and the refractive index of core 2 would decrease. Consequently, the propagation constants of core 1 and core 2 would increase and decrease, respectively, as shown in Fig.5. The effective refractive index of core 1 and core 2 would increase and decrease, respectively, and the difference of effective index $\Delta \phi$ would increase. According to Eq.(2), the peak wavelength would move towards the longer wavelength. When core 2 is in the out layer and core 1 is in the inner layer, Fig.5 also shows the variation of the propagation constants of dual cores. 


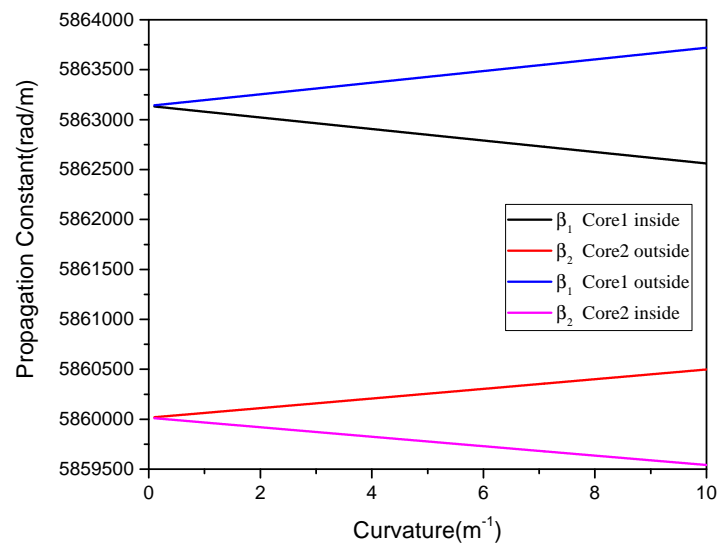

Figure 5: Propagation constants against curvature with two case; one of the case is core 1 in the out layer (blue line) and core 2 in the inner layer (pink line), another case is core 1 in the inner layer (black line) and core 2 in the out layer (red line).

The propagation constants of core 1 and core 2 would decrease and increase with the variation of the curvature, respectively. With the same theory as above, the effective refractive index of core 1 and core 2 would decrease and increase, respectively. The difference of effective index $\Delta \phi$ would increase, which causes the peak wavelength blue shift.

\section{Experiment and discussion}

In the experiment, the fabrication process of the proposed fiber sensor has two steps. First, a SMS structure is fabricated with the MMF equal to twice length of the single MMF, and the SMS structure is cut in the axial center of the MMF. The second step is that the TCF is spliced between the MMFs to realize the proposed configuration. The transmitted spectrum is shown in Fig.6. It can be seen from Fig.6 that the extinction ratio is about $15 d B$, and remarkable repeatability of the MZI comb filter can be achieved. 
The schematic of the cascaded device and the instruments utilized in the experiment are shown in Fig.7. A broad band source (BBS) is employed as the light source. The transmission spectrum of the sensor head is detected by an optical spectrum analyzer (OSA).

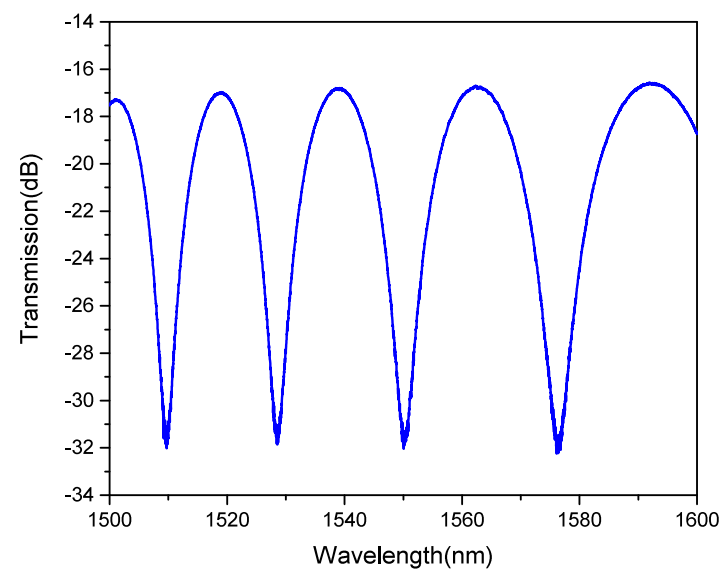

Figure 6: Transmission spectrum of the TCF with MMFS.

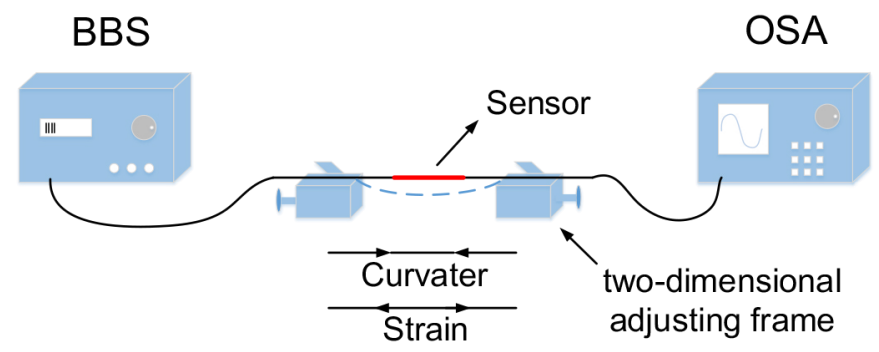

Figure 7: Schematic diagram of the experiment setup.

Two translation stages are used to change the curvature of the device, and the initial distance between the two translation stages is $500 \mathrm{~mm}$. The relationship between the curvature of the structure and the parameters can 
be calculated as

$$
c=\frac{1}{R} \cong \sqrt{\frac{24 d}{L_{0}^{3}}}
$$

where $L_{0}$ and $d$ are the initial distance and the movement distance between the two translation stages, respectively.

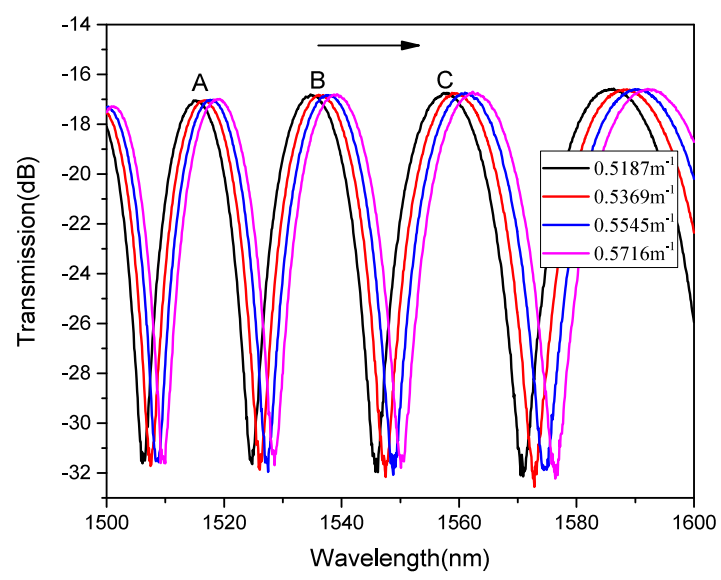

Figure 8: transmission spectra of the fiber sensor for various curvature.

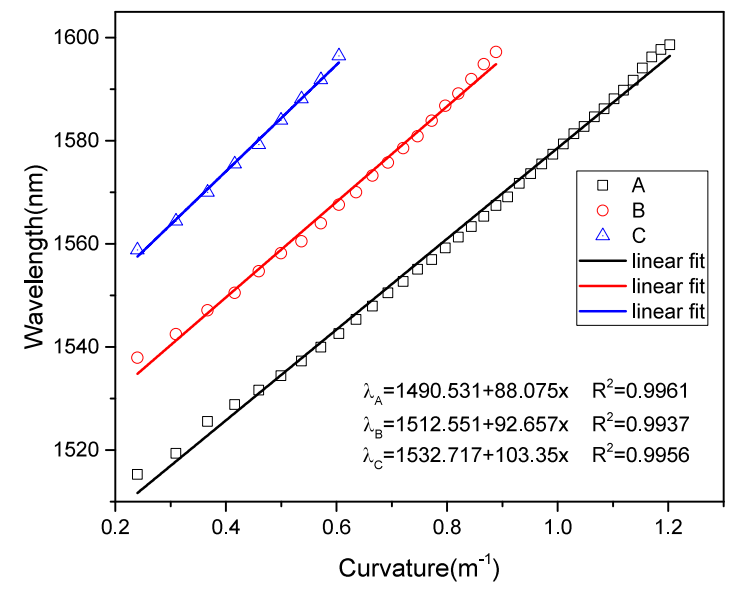

Figure 9: The relationship between the peak wavelength and the curvature.

Fig.8 shows the transmission spectra of the sensor under different bend- 
ing curvatures. With the increase of the curvature, the peak wavelength shift towards the longer wavelength, which agrees with the theoretical analysis above. By the linear fitting method for the peak wavelengths, the relationship between the peak wavelength and the curvature is shown in Fig.9. In the limited optical spectral ranges, the curvature sensitivity of the sensor is $88.075 \mathrm{~nm} / \mathrm{m}^{-1}$ for the wavelength around $1520 \mathrm{~nm}$ ranging from $0.24 \mathrm{~m}^{-1}$ to $1.2 \mathrm{~m}^{-1}, 92.657 \mathrm{~nm} / \mathrm{m}^{-1}$ for the wavelength around $1540 \mathrm{~nm}$ ranging from $0.24 \mathrm{~m}^{-1}$ to $0.89 \mathrm{~m}^{-1}$, and $103.35 \mathrm{~nm} / \mathrm{m}^{-1}$ for the wavelength around 1560 $\mathrm{nm}$ ranging from $0.24 \mathrm{~m}^{-1}$ to $0.6 \mathrm{~m}^{-1}$, respectively. It is also found from Fig.9 that the curvature sensitivity of the sensor increases with the increase of the wavelength, which can be explained by

$$
\begin{gathered}
\lambda_{m}^{\prime}=\frac{(\Delta n+\delta n) L}{m} \\
\delta \lambda_{m}=\lambda_{m}^{\prime}-\lambda_{m}=\frac{\delta n}{\Delta n} \lambda_{m}
\end{gathered}
$$

where $\delta n$ is a small change in value of $\Delta n$ by applying the curvature to the sensor. $\lambda_{m}^{\prime}$ is the corresponding peak wavelength, and $\delta \lambda_{m}$ is the small change in value of the peak wavelength $\lambda_{m}$. It is found that longer wavelength gives larger increase in $\delta \lambda_{m}$ for a fixed increase in the $\delta n$ in Eq.(8). Since $\delta n$ is corresponding to the change of the curvature, therefore, long wavelength gives larger wavelength shift for a fixed increase in the curvature.

The fiber sensor also can be used to measure the strain. The two translation stage was adjusted with a step of $0.1 \mathrm{~mm}$, which is equal to a strain step of $200 \mu \varepsilon$. The transmission spectra of the sensor under different strain is shown in Fig.10, in which the transmission spectrum of the sensor shifts to the short wavelength with the strain ranges from $0 \mu \varepsilon$ to $1400 \mu \varepsilon$. 


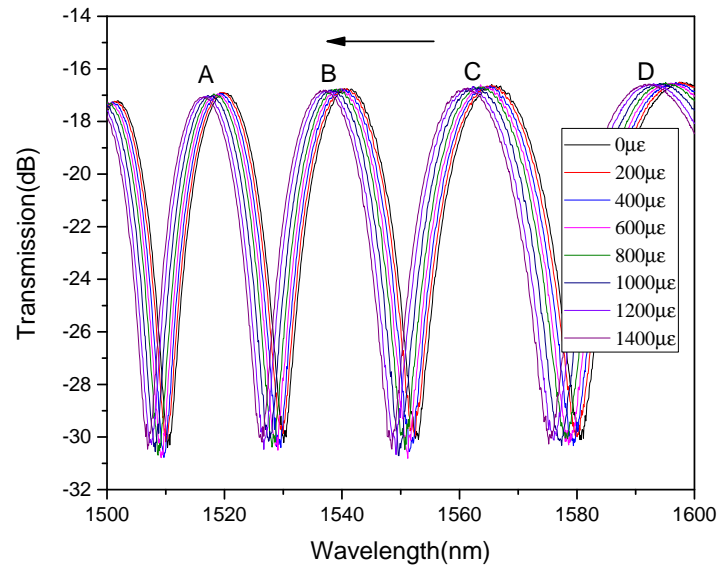

Figure 10: transmission spectra of the fiber sensor for various strain.

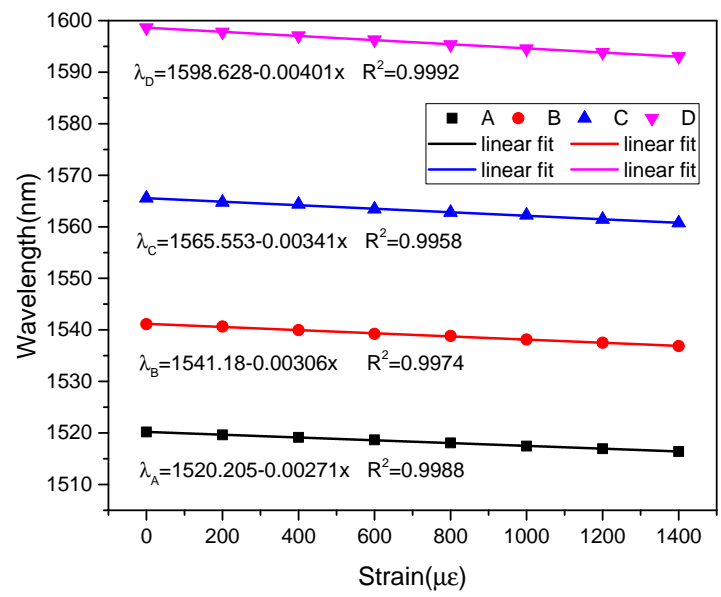

Figure 11: The relationship between the peak wavelength and the strain . 
When external strain is applied to the TCF, According to elastic-optic effect, the refractive index of the dual cores are decreased, and the effective refractive index of both cores are decreased. The decreasing magnitude for the large core is more than that for the small core. This results in a decrease in $\Delta \phi$, and the peak wavelength shift towards the shorter wavelength with an increase in the strain. In Fig.11 the linear fitting result shows that the strain sensitivity is $-2.71 \mathrm{pm} / \mu \varepsilon$ around the resonance wavelength of $1520 \mathrm{~nm},-3.06$ $\mathrm{pm} / \mu \varepsilon$ around the resonance wavelength of $1540 \mathrm{~nm},-3.41 \mathrm{pm} / \mu \varepsilon$ around the resonance wavelength of $1565 \mathrm{~nm}$, and $-4.01 \mathrm{pm} / \mu \varepsilon$ around the resonance wavelength of $1580 \mathrm{~nm}$ in the range from $0 \mu \varepsilon$ to $1400 \mu \varepsilon$, respectively.

Since the different resonance wavelengths have different sensitivities towards curvature and strain, the variations of curvature and strain can be discriminated, and can be calculated using coefficient matrix as [13]

$$
\left[\begin{array}{c}
\Delta C \\
\Delta S
\end{array}\right]=\frac{1}{D}\left[\begin{array}{cc}
K_{S, C} & -K_{S, B} \\
-K_{C, C} & K_{C, B}
\end{array}\right]\left[\begin{array}{c}
\Delta \lambda_{B} \\
\Delta \lambda_{C}
\end{array}\right]
$$

where $\Delta \mathrm{C}$ and $\Delta \mathrm{S}$ are the variation of curvature and strain, respectively. $\mathrm{D}=K_{S, C} K_{C, B}-K_{S, B} K_{C, C}, K_{C, B}$ and $K_{S, B}$ are the curvature and strain sensitivities of peak B, respectively; $K_{C, C}$ and $K_{S, C}$ are the curvature and strain sensitivities of peak $\mathrm{C}$, respectively. $\Delta \lambda_{B}$ and $\Delta \lambda_{C}$ are the wavelength shift of peak B and peak C, respectively. Therefore, Eq.9 can be rewritten with the experimental results, the matrix can be given as

$$
\left[\begin{array}{l}
\Delta C \\
\Delta S
\end{array}\right]=\frac{1}{0.00029}\left[\begin{array}{ll}
-0.00341 & 0.00306 \\
-103.35 & 92.657
\end{array}\right]\left[\begin{array}{l}
\Delta \lambda_{B} \\
\Delta \lambda_{C}
\end{array}\right]
$$




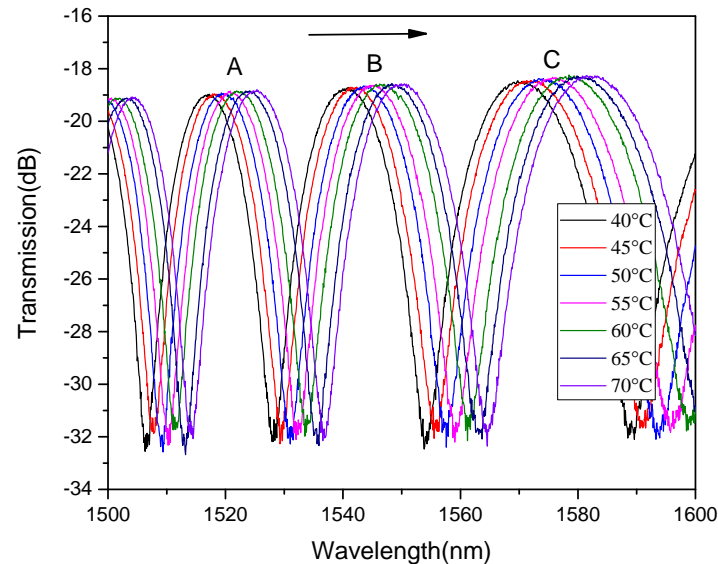

Figure 12: transmission spectra of the fiber sensor for various temperature.

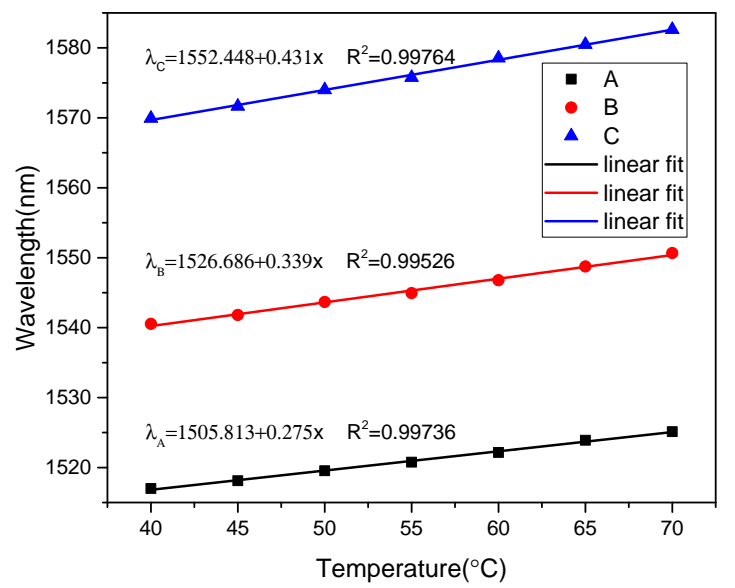

Figure 13: The relationship between the peak wavelength and the temperature. 
In the experiment, the response of the proposed fiber sensor to temperature has been investigated. Fig.12 shows the resonant wavelength shifting with the different temperature. It could be seen that with the temperature increasing the resonant wavelength shifts to the short wavelength. The linear relationship between the temperature and the resonant wavelength is shown in Fig.13, and the temperature sensitivity is $0.275 \mathrm{~nm} /{ }^{\circ} \mathrm{C}$ around the resonance wavelength of $1520 \mathrm{~nm}, 0.339 \mathrm{~nm} /{ }^{\circ} \mathrm{C}$ around the resonance wavelength of $1545 \mathrm{~nm}$, and $0.431 \mathrm{~nm} /{ }^{\circ} \mathrm{C}$ around the resonance wavelength of $1575 \mathrm{~nm}$ in the range from $40{ }^{\circ} \mathrm{C}$ to $70{ }^{\circ} \mathrm{C}$, respectively. Consequently, this curvature sensitivity is almost four times higher than that of the sensors[9]. This sensitivity is approximately five times that of the curvature sensor by using two peanut-shape structure[8], and compared with other fiber sensors it is very $\operatorname{high}[7,12,13]$.

\section{Conclusion}

In this paper, we proposed and experimentally demonstrated an optical fiber sensor based asymmetrical TCF and MMF. By applying the coupledmode theory and equivalent refractive index model, we theoretically analyze the uncoupled feature of the TCF and the relationship between peak wavelength and the curvature. Two segments of MMFs are used as beam splitter and combiner to form the in-fiber MZI. The extinction ratio of the MZI is about $15 d B$. In the experiment, the curvature sensitivity of the device can be achieved as high as $103.35 \mathrm{~nm} / \mathrm{m}^{-1}$ ranging from $0.24 \mathrm{~m}^{-1}$ to $0.6 \mathrm{~m}^{-1}$, and the sensitivity of strain is $-4.01 \mathrm{pm} / \mu \varepsilon$ in the range from $0 \mu \varepsilon$ to 1400 $\mu \varepsilon$. The simultaneous detection of the curvature and strain can be realized 
by substituting the sensitivities to coefficient matrix. The temperature sensitivity is $0.431 \mathrm{~nm} /{ }^{\circ} \mathrm{C}$ in the range from $40{ }^{\circ} \mathrm{C}$ to $70{ }^{\circ} \mathrm{C}$. The result shows that the sensor is suited for curvature, strain and temperature measurement. In conclusion, the proposed sensor is characterized by compact, structural stability, easy-fabrication and high sensitivity, and it has a great potential to be applied in optical sensing system.

\section{Acknowledgments}

This work was supported by the National Science Foundation for Distinguished Young Scholars of China (Grant No. 61525501).

\section{References}

[1] O. F. ao, J. Viegas, P. Caldas, J. L. Santos, F. M. Araújo, L. A. Ferreira, F. Farahi, All-fiber mach-zehnder curvature sensor based on multimode interference combined with a long-period grating, Opt. Lett. 32 (21) (2007) 3074-3076. doi:10.1364/OL.32.003074.

[2] L. Shao, A. Laronche, M. Smietana, P. Mikulic, W. J. Bock, J. Albert, Highly sensitive bend sensor with hybrid long-period and tilted fiber bragg grating, Opt. Commun. 283 (13) (2010) 2690 - 2694. doi:http://dx.doi.org/10.1016/j.optcom.2010.03.013.

[3] H. J. Patrick, G. M. Williams, A. D. Kersey, J. R. Pedrazzani, A. M. Vengsarkar, Hybrid fiber bragg grating/long period fiber grating sensor for strain/temperature discrimination, IEEE Photon. Technol. Lett. 8 (9) (1996) 1223-1225. doi:10.1109/68.531843. 
[4] Y. Jin, C. Chan, X. Dong, Y. Zhang, Temperature-independent bending sensor with tilted fiber bragg grating interacting with multimode fiber, Opt. Commun. 282 (19) (2009) 3905 - 3907. doi:http://dx.doi.org/10.1016/j.optcom.2009.06.058.

[5] Y. Huang, T. Guo, C. Lu, H. Y. Tam, Vcsel-based tilted fiber grating vibration sensing system, IEEE Photon. Technol. Lett. 22 (16) (2010) 1235-1237. doi:10.1109/LPT.2010.2052797.

[6] X. Dong, Y. Liu, L. Y. Shao, J. Kang, C. L. Zhao, Temperature-independent fiber bending sensor based on a superimposed grating, IEEE Sens. J. 11 (11) (2011) 3019-3022. doi:10.1109/JSEN.2011.2157124.

[7] Y. Gong, T. Zhao, Y. J. Rao, Y. Wu, All-fiber curvature sensor based on multimode interference, IEEE Photon. Technol. Lett. 23 (11) (2011) 679-681. doi:10.1109/LPT.2011.2123086.

[8] H. Gong, X. Yang, K. Ni, C. L. Zhao, X. Dong, An optical fiber curvature sensor based on two peanut-shape structures modal interferometer, IEEE Photon. Technol. Lett. 26 (1) (2014) 22-24. doi:10.1109/LPT.2013.2288978.

[9] L. Niu, C.-L. Zhao, H. Gong, Y. Li, S. Jin, Curvature sensor based on two cascading abrupt-tapers modal interferometer in single mode fiber, Opt. Commun. 333 (2014) 11 - 15. doi:http://dx.doi.org/10.1016/j.optcom.2014.07.036. 
[10] L. Ma, Y. Qi, Z. Kang, S. Jian, All-fiber strain and curvature sensor based on no-core fiber, IEEE Sens. J. 14 (5) (2014) 1514-1517. doi:10.1109/JSEN.2014.2298553.

[11] C. Gouveia, P. A. S. Jorge, J. M. Baptista, O. Frazao, Temperatureindependent curvature sensor using fbg cladding modes based on a core misaligned splice, IEEE Photon. Technol. Lett. 23 (12) (2011) 804-806. doi:10.1109/LPT.2011.2138125.

[12] H. Gong, H. Song, X. Li, J. Wang, X. Dong, An optical fiber curvature sensor based on photonic crystal fiber modal interferometer, Sens. Actuators, A 195 (2013) 139 - 141. doi:http://dx.doi.org/10.1016/j.sna.2013.02.022.

[13] Y. Zhou, W. Zhou, C. C. Chan, W. C. Wong, L.-Y. Shao, J. Cheng, X. Dong, Simultaneous measurement of curvature and temperature based on pcf-based interferometer and fiber bragg grating, Opt. Commun. 284 (24) (2011) 5669 - 5672. doi:http://dx.doi.org/10.1016/j.optcom.2011.08.048.

[14] B. Dong, J. Hao, Z. Xu, Temperature insensitive curvature measurement with a core-offset polarization maintaining photonic crystal fiber based interferometer, Opt. Fiber. Technol. 17 (3) (2011) 233 - 235. doi:http://dx.doi.org/10.1016/j.yofte.2011.02.008.

[15] K. Ni, T. Li, L. Hu, W. Qian, Q. Zhang, S. Jin, Temperatureindependent curvature sensor based on tapered photonic crystal 
fiber interferometer, Opt. Commun. 285 (24) (2012) 5148 - 5150. doi:http://dx.doi.org/10.1016/j.optcom.2012.08.037.

[16] H. Zou, S. Lou, G. Yin, A wavelength-tunable fiber laser based on a twin-core fiber comb filter, Opt. Laser Technol. 45 (2013) 629 - 633. doi:http://dx.doi.org/10.1016/j.optlastec.2012.05.020.

[17] K. Nagano, S. Kawakami, S. Nishida, Change of the refractive index in an optical fiber due to externalforces, Appl. Opt. 17 (13) (1978) 20802085. doi:10.1364/AO.17.002080. 